\title{
Les compétences de l'ingénieur hydraulicien de demain dans le domaine des aménagements fluviaux et maritimes
}

\author{
G. Nicollet - EDF, Laboratoire National d'Hydraulique, Chatou \\ Ch. Puyo - SOGREAH, Grenoble \\ P. Robert - EDF, Division Technique Générale, Grenoble \\ R. Pinatel et E. Tormos - Compagnie Nationale du Rhône, Lyon
}

Jusqu'à une époque récente, les aménagements fluviaux et maritimes, désignés ci-après par "les aménagements", devaient satisfaire en priorité l'objectif fixé, et c'était la tâche principale de l'Ingénieur Hydraulicien, intervenant dans leur conception, leur réalisation et leur exploitation.

Depuis, force est de constater que les ressources en eau ne sont pas inépuisables, alors que les besoins et donc la demande ne cessent d'augmenter. Ainsi, et bien que l'Hydraulique proprement dite garde toute son importance, les autres facteurs intervenant dans les tâches de conception, de réalisation, et d'exploitation tels l'Economie, l'Environnement, la Socio-Economie, etc... prendront de plus en plus d'importance.

Devant ce constat, quel comportement peut et doit avoir l'Ingénieur? Pour en faire l'analyse, nous avons cherché à avoir l'opinion d'un certain nombre d'Ingénieurs des trois établissements que nous représentons (Electricité de France, SOGREAH, Compagnie Nationale du Rhône).
Pour cela, nous leur avons soumis un questionnaire dont le modèle est donné en annexe. Ce dernier comprend deux séries de questions; la première permettant de faire le constat de la situation actuelle, la deuxième définissant l'évolution future du métier et les compétences que devra acquérir l'Ingénieur pour accomplir sa mission.

Il est évident que les résultats de cette enquête ne permettent pas de définir de manière exhaustive les qualités et compétences nécessaires que devra avoir l'Ingénieur de demain dans le domaine des aménagements fluviaux et maritimes. Ils peuvent cependant constituer une base intéressante de départ pour la réflexion à mener au cours de ce colloque.

Le dépouillement des réponses recueillies amène une remarque préalable importante: ce questionnaire a été reçu, de la part d'un nombre non négligeable d'Ingénieurs consultés, avec un certain embarras. En particulier, certains ont contesté l'appellation "Hydraulicien", ne voyant pas les spécificités qu'il devait avoir. Les résultats sont présentés dans les paragraphes suivants.

\section{The skills of tomorrow's hydraulics engineer in the river and maritime engineering field}

Up until quite recently, hydraulic schemes had in priority to meet the targets fixed, and this was the main task of the hydraulics engineer involved in the design, construction and operation of river engineering schemes, for example. Nowadays, the manner in which a development scheme is designed, constructed, or operated, has changed quite considerably. Although the hydraulics aspect is obviously still of great importance, if only because water resources can never be considered inexhaustible and the demand for water is always increasing, greater allowance must now be made for other factors such as Economics, Environment, Socio-Economics, etc. The hydraulics engineer in charge of a project cannot afford to ignore these aspects. 


\section{Le rôle et les compétences de l'Ingénieur Hydrau- licien actuellement}

Actuellement, l'Ingénieur intervient dans la conception la réalisation et l'exploitation des aménagements sur les problèmes propres à sa spécialité, c'est-à-dire :

- Hydrologie,

- Morphologie,

- Modélisation mathématique et modélisation physique,

- Dimensionnement des ouvrages (prises d'eau, évacuation, etc...),

- Hydraulique en général,

tandis que sont traitées par les spécialistes correspondants les autres disciplines qui interviennent, à savoir :

- Géologie,

- Géotechnique,

- Pédologie,

- Hydrogéologie,

- Topographie,

- Génie Civil,

- Electromécanique,

- Automatisme,

- Environnement (Biologie, Agriculture, Urbanisme),

- Economie (et Politique),

- etc...

Cependant, l'Ingénieur ne doit pas méconnaître complètement ces divers aspects, qu'il soit intégré comme spécialiste de l'hydraulique des ouvrages dans une équipe pluridisciplinaire ou responsable d'un projet faisant intervenir plusieurs spécialistes dont il aura à coordonner les études partielles.

Sa formation doit donc le préparer à acquérir un minimum de compétences dans ces disciplines connexes.

\section{L'évolution dans la conception, la réalisation et l'exploitation des aménagements}

Les préoccupations concernant l'environnement, si elles n'ont pas toujours été le principal souci de l'Ingénieur, sont aujourd'hui prises en compte au niveau même des procédures administratives.

Une étude d'impact s'ajoute désormais aux études techniques, économiques et financières. Son but est de respecter au mieux le milieu et de prévoir, si nécessaire, des mesures compensatoires pour limiter les conséquences dommageables éventuelles du projet. En outre, dans certains cas, le dossier peut être complété par une étude sur les aspects architecturaux et paysagers.

D'autre part, et comme nous l'avons dit plus haut, les ressources naturelles en eau n'étant pas inépuisables et les besoins étant de plus en plus importants, l'Ingénieur en charge du projet a le devoir d'aboutir à la solution conciliant au mieux toutes les contraintes (Economie, Dimensionnement, Environnement, Socio-Economie, Loisirs,...). Ceci est d'autant plus nécessaire que la pression de l'opinion publique au regard de l'eau, souvent relayée par les médias, est très forte.
En outre, les procédures administratives prévoient des concertations préalables avec les riverains concernés (Collectivités locales et individus) et les Administrations.

Ainsi, lorsque le projet est complètement instruit, on peut dire que l'Ingénieur a conçu un aménagement intégré.

Cette concertation quasi permanente prendra de plus en plus d'importance et obligera l'Ingénieur à évaluer tous les impacts avec une très grande précision. Ceci nécessitera entre autres la mise en œuvre de modèles physiques, mais surtout mathématiques de plus en plus sophistiqués, les moyens informatiques, étant devenus un des outils privilégiés de l'Ingénieur. Il y aura nécessité également de mettre en place d'un dialogue permanent permettant aux différents partenaires de communiquer entre eux. Ce dialogue se fera non seulement au cours de réunions et d'exposés, mais aussi grâce à des notes et rapports dont le contenu sera facilement accessible au destinataire.

Par ailleurs, la prise en compte des risques industriels et liés à l'environnement oblige de plus en plus les Ingénieurs à travailler dans le cadre des procédures «d'Assurance Qualité ", ce qui implique un formalisme et une rigueur particuliers dans la réalisation des études.

Les aménagements intégrés recourront de plus en plus à des équipes pluridisciplinaires et feront aussi appel à des financements d'origines diverses, de telle sorte que la concertation devra se poursuivre en phases de réalisation et d'exploitation.

\section{Les compétences demandées à l'Ingénieur du futur}

Les expériences des décennies récentes ont montré que le métier de l'Ingénieur a évolué; quiconque ayant une certaine ancienneté n'a qu'à se rappeler les activités de son début de carrière.

Il est à peu près évident que cette évolution ne peut que se poursuivre et que le meilleur atout de l'Ingénieur pour faire face est d'avoir acquis une solide formation de base (Mathématiques, Mécanique, Physique, Mécanique des Fluides, Analyse Numérique,...).

La formation initiale suivie ne doit pas impliquer obligatoirement une activité professionnelle exclusivement liée à cette spécialité. L'Ingénieur devra posséder la faculté de pouvoir s'intégrer dans une équipe, puis tout au long de sa carrière de pouvoir suivre les évolutions de son métier, voire s'adapter à des activités complètement différentes.

Les compétences qui seront demandées à l'Ingénieur Hydraulicien devront l'amener à développer son sens de l'observation du terrain et de l'analyse critique des données recueillies.

La confrontation des résultats de calculs obtenus à partir de logiciels de plus en plus performants, avec des mesures in-situ ou en laboratoire, sera plus que jamais nécessaire, en particulier dans le domaine de la sédimentologie et du transport solide.

Outre le sens physique et les connaissances classiques du généraliste, l'Ingénieur aura besoin d'une excellente aptitude à l'informatique. Il devra comprendre parfaitement les principes sur lesquels sont bâtis les logiciels qu'il utilise. 
Les impératifs de "l'Assurance Qualité " impliqueront de la part de l'Ingénieur de la rigueur et le respect d'un formalisme contraignant.

On peut alors se poser la question suivante : l'Ingénieur Hydraulicien dit-il avoir des compétences particulières?

La réponse n'est pas évidente :

L'Ingénieur Hydraulicien dans le domaine des aménagements fluviaux et maritimes doit avoir les compétences de tout ingénieur chargé d'un aménagement quel qu'il soit.

Mais de plus, et parce que les aménagements fluviaux et maritimes sont très liés à leur environnement naturel, il doit être un homme de terrain capable d'intégrer toutes les composantes du projet.

Les connaissances acquises en hydraulique lui seront indispensables pour vérifier et justifier par le calcul ce que l'observation, l'expérimentation et l'imagination l'auront conduit à projeter. Mais elles ne suffiront pas pour faire de tout Ingénieur Hydraulicien un bon aménageur.
En effet, l'Ingénieur devra être capable de mettre ses compétences au service de l'équipe pluridisciplinaire nationale ou multinationale chargée du projet. Pour cela, il faudra qu'il puisse communiquer facilement au sein de cette équipe en utilisant les langages adéquats, à savoir : tous les langages techniques traditionnels, langues étrangères, langage écologique, langage politique, langage économique, etc...

L'état du marché dans le domaine des aménagements fluviaux et maritimes ne semble pas devoir être en expansion dans les années futures et les Entreprises de ce secteur ont plutôt tendance à travailler à effectif constant.

Les besoins en Ingénieurs Hydrauliciens dans ces disciplines ne seront donc pas croissants et se limiteront probablement au maintien du potentiel existant.

Dans ce contexte, les Ingénieurs chargés de ces aménagements devront faire preuve d'encore plus d'enthousiasme et d'imagination et devront savoir communiquer pour promouvoir les projets.

\section{QUESTIONNAIRE \\ L'INGÉNIEUR HYDRAULICIEN DEMAIN ÉVOLUTION DU MÉTIER}

- Quelles sont à votre avis les différentes phases de CONCEPTION d'un aménagement hydraulique :

- Aménagement à buts multiples (usine, barrage, canaux de navigation, agriculture,...).

- Aménagement de rivière.

- Pour chacune de ces phases, quelles sont les disciplines en jeu (géologie, géotechnique, hydraulique, électricité, électromécanique, environnement, économie, agriculture,,.. ?).

- Actuellement, comment doivent intervenir les Ingénieurs Hydrauliciens dans ces différentes phases et quelles compétences doivent-ils avoir?

- Dans l'avenir :

a) Comment voyez-vous l'évolution dans la conception des aménagements?

b) Quelles compétences seront nécessaires pour l'Ingénieur Hydraulicien?

Pouvez-vous également répondre aux mêmes questions que ci-dessus pour la "RÉALISATION ", "L'EXPLOITATION » et la mise en œuvre des « MESURES et EXPÉRIMENTATION » concernant ces mêmes aménagements. 